\title{
La paz y el desarrollo sostenible en el campo educativo, una relación visible o invisible
}

\section{Peace and Sustainable Development in Education, a Visible or Invisible Relationship}

Damian Filut ${ }^{*}$

Centro Internacional de Formación Profesional en Educación de Mashav Agencia Israelí de Cooperación Internacional para el Desarrollo, Israel http://orcid.org/0000-0002-2443-655X

Artículo de investigación

Fecha de recepción: 27 de enero de 2020

Fecha de aceptación: 14 de abril de 2020

\section{Para citar este artículo:}

Filut, D. (2020). La paz y el desarrollo sostenible en el campo educativo, una relación visible o invisible. Campos en Ciencias Sociales, 8(2), 133-158. DoI: https://doi. org/10.15332/25006681/6015

Experto internacional en educación para el desarrollo, licenciado en Estudios Internacionales, doctorando por la Universidad Hebrea de Jerusalén, magíster en Estudios Europeos. Su interés investigativo se centra en las políticas de cooperación internacional para el desarrollo. Vicedirector del Centro mashav de Capacitación Internacional para la Educación, Agencia Israelí de Cooperación Internacional para el Desarrollo. Jerusalén, Israel. Correo electrónico: damian@metc.org.il 


\section{RESUMEN}

Los conceptos paz y desarrollo sostenible hacen parte integral del diálogo en el campo del desarrollo internacional y también se ven reflejados en los programas de estudio de varios países. Esta investigación busca identificar la relación entre estos dos conceptos en el marco de la educación y la manera como se evidencia dicha relación en la práctica educativa. Se toma el caso de la educación para el desarrollo sostenible en Israel, que cuenta con un programa nacional en el Ministerio de Educación. Teniendo como referencia a la Red Verde - una de las organizaciones a cargo de la formación docente en esta materia—, se busca entender esta relación y su carácter. Los resultados obtenidos son producto del análisis de documentos oficiales israelíes para una primera contextualización, y cuestionarios en línea y entrevistas semiestructuradas a capacitadores de educación para el desarrollo sostenible. Estos nos muestran la gran importancia del contexto en el momento de tratar un tema como la paz y cómo la educación para la sustentabilidad podría tener un lugar fundamental para conseguirla.

Palabras clave: educación para el desarrollo sostenible, educación para la paz, formación de docentes.

\section{Abstract}

The concepts of peace and sustainable development are an integral part of dialogue in the field of international development and are also contained in the curricula of several countries. This research paper seeks to identify the relationship between these two concepts in the framework of education and how this relationship is evinced in educational practice. The selected case for this research is that of education for sustainable development in Israel, which has a national program in the Ministry of Education. Taking as reference the Green Network - one of the organizations in charge of teacher training in this field—, it seeks to understand this relationship and its nature. The results obtained are the result of contents analysis of official Israeli documents, for an initial contextualization, and online questionnaires and semi-structured interviews with education for sustainable development trainers. The results show us the great importance of the context in dealing with a issue such as peace and how education for sustainability could play a fundamental role in its achievement.

Keywords: education for sustainable development, education for peace, teacher training. 


\section{Agradecimientos}

Esta investigación fue desarrollada con el apoyo de la Red Verde, una organización israelí encargada de capacitar a los docentes en educación para el desarrollo sostenible dentro del programa de Educación para la Sostenibilidad del Ministerio de Educación de Israel.

\section{INTRODUCCIÓN}

Los conceptos paz y desarrollo forman parte de la historia de la humanidad. Las relaciones humanas entre individuos y comunidades se pueden conocer a través de la sucesión de estos dos conceptos. La humanidad se encuentra en procesos continuos en busca de paz, para así fomentar procesos efectivos de desarrollo. En el siglo xx estos procesos se vieron reflejados en los sistemas educativos, que tenían a su cargo formar las futuras generaciones. Es decir, estas prácticas y contenidos se impartieron e incluyeron en los programas educativos del mundo. Fue así como aparecieron los programas de educación para la paz y desarrollo sostenible.

Los programas de educación para la paz son previos a los de educación para el desarrollo sostenible, los cuales son aún relativamente nuevos. Estos aparecieron formalmente en la década de 1990, con la creación de dicho concepto (Harris, 2004; Goldman, Yavetz y Pe’er, 2006; Bajaj y Chiu, 2009). De aquí que surge la pregunta: ¡en estos procesos educativos existe una relación entre los conceptos paz y desarrollo, como se ve en los acuerdos y declaraciones internacionales? Conjuntamente es interesante observar la naturaleza es esta relación, si es visible o invisible; visible haciendo referencia a la existencia de esta relación en los programas y las prácticas, e invisible lo contrario - la inexistencia de la relación entre los conceptos paz y desarrollo-.

Esta investigación escoge un contexto particular por su situación y características. El caso de análisis es el programa de educación para la sustentabilidad del Ministerio de Educación de Israel. El programa fue analizado a través de documentos del Ministerio y entrevistas y cuestionarios realizados a un grupo de capacitadores de docentes de una de las dos organizaciones que forman docentes en esta temática, la Red Verde. 


\section{Paz y desarrollo desde la comunidad internacional}

Para entender la relación entre estos dos conceptos y su proyección en el sistema educativo de hoy, se señala la manera en que estos se proyectaron desde la segunda mitad del siglo xx. A continuación, se describe el desarrollo desde el nivel internacional, siendo este un punto de referencia para muchos países en estas áreas.

Un primer punto de referencia es La Carta de las Naciones Unidas, firmada en 1945, que establece, en su primer artículo, como propósito primordial "Mantener la paz y la seguridad internacional" (Naciones Unidas, 1945, p. 3). Esta elección deja en claro las intenciones de este organismo. Si en el pasado estos conceptos hicieron parte de la visión y misión de organismos internacionales, en las últimas décadas estos conceptos se han convertido en objetivos tangibles y operativos para su logro (Filut, 2018). De este documento se entendiera que la paz entre las naciones y entre los seres humanos, ya desde la segunda mitad del siglo $\mathrm{xx}$, se convirtió en un requisito/ necesidad para el desarrollo de la humanidad. Luego de guerras que destruyeron a países por completo y dejaron al mundo en una situación de crisis, el hincapié en esta idea no debería llamar la atención (Surendra, 2014).

En paralelo, se da un proceso a través del cual un nuevo concepto entra a la retórica internacional. En los últimos 30 años, el desarrollo sostenible se ha convertido en uno de los conceptos más utilizados en el campo del desarrollo internacional. Este cobra un valor real en 1987 con el reporte de Bruntland, en el cual, de manera formal, se definió como el desarrollo "que satisface las necesidades del presente sin comprometer las necesidades de las futuras generaciones." (World Commission on Environment and Development, 1987, p. 16). En este momento, su caracterización y adopción se vieron reflejadas en una serie de cumbres internacionales (Cumbre de Río 1992, Cumbre del Milenio 2000, Cumbre Mundial sobre Desarrollo Sostenible 2002, Conferencia Río + 20 2012, Cumbre de Desarrollo Sostenible de la onu 2015), a través de las cuales este concepto se tradujo en indicadores y variables que pueden ser evaluados e implementados por los países miembros (Agudelo, 2018; Filut, 2018).

Tanto los Objetivos del Mileno como los Objetivos del Desarrollo Sostenible son fiel reflejo del lugar que la paz tiene en estos esquemas. En el preámbulo de la resolución Transformar nuestro mundo: la Agenda 2030 para el Desarrollo Sostenible del 2015 
se establecieron los 17 Objetivos del Desarrollo Sostenible y se enunciaron cinco conceptos en los aparece la paz: "Estamos decididos a propiciar sociedades pacíficas, justas e inclusivas que estén libres del temor y la violencia. No puede haber desarrollo sostenible sin paz, ni paz sin desarrollo sostenible” (Naciones Unidas, 2015, p. 2).

Con la puesta en práctica de los productos de estas cumbres y con base en este nuevo concepto de desarrollo, las Naciones Unidas, en colaboración con los países miembro, reafirmó la importancia de la paz en los procesos de desarrollo.

\section{Objetivos de la investigación}

Esta investigación pretende identificar qué tipo de relación existe entre estos dos conceptos, paz y desarrollo sostenible, en el sector educativo, en el que las prácticas educativas en ambas temáticas toman lugar. Dos cuestiones se desprenden de esta relación y se analiza el caso del programa de educación en Israel.

En la realidad internacional, antes presentada, el concepto de paz se encuentra integrado con el de desarrollo sostenible. Por lo tanto, el primer objetivo de esta investigación es comprender si esto se traslada al escenario de los países o se mantiene únicamente en el marco internacional. Quedan claras las limitaciones de analizar un caso único, pero se espera que los resultados de esta investigación contribuyan a futuros trabajos comparativos con las situaciones de otros países.

En segunda instancia, se pretende entender si esta relación en el marco educativo existe en la teoría (documentos oficiales) y/o en la práctica (programas educativos). Luego de un primer análisis de contenido, se entrevistaron educadores del sistema educativo israelí para comprender qué dimensiones relacionadas con la educación para la paz hacen parte de su práctica educativa.

\section{La educación para la sustentabilidad en Israel}

El programa de Educación para la Sustentabilidad del Ministerio de Educación en Israel es una iniciativa desarrollada en conjunto con el Ministerio del Medio 
Ambiente, que comenzó como un programa de capacitación institucional para el desarrollo del personal docente. El hecho de que sea un programa en cooperación con el Ministerio del Medio Ambiente indica que su orientación es, sobre todo, ambiental (Ministerio de Educación de Israel, 2019).

El programa de Educación para la Sustentabilidad en Israel está diseñado para el personal docente en jardines de infantes, escuelas primarias y secundarias. Uno de sus objetivos es guiar un proceso de certificación de instituciones ecológicas en estos espacios educativos (Ministerio de Educación de Israel, 2012).

La señora Hani Peleg, directora del programa de Educación para la Sustentabilidad en Israel del Ministerio de Educación, explica que el programa funciona en cooperación con los organismos verdes: la Red Verde y la Sociedad para la Protección de la Naturaleza. Esta particularidad deja en claro que en el sistema educativo israelí se trabaja con la colaboración de organismos no gubernamentales (con supervisión ministerial), bajo la premisa de que estos tienen conocimientos importantes que aportar. En segundo lugar, para entender mejor la orientación del programa es fundamental observar a los entes responsables de gran parte de los contenidos (H. Peleg, comunicación personal, 28 de diciembre de 2019). Finalmente, el hecho de llamar a estas organizaciones verdes, fortalece el argumento de su componente ambiental.

\section{¿Cómo funciona el programa?}

La participación en el programa es un proceso decidido paso a paso por la escuela (como institución). No se trata de una capacitación de un docente, sino de un proceso integral que la institución experimenta. A diferencia de otras capacitaciones que se dictan en centros de formación docente en servicio del Ministerio de Educación, la formación profesoral en este programa se imparte en la institución misma. Un 80 \% de los docentes debe participar en los niveles de prescolar y primaria; y en los niveles de secundaria, un mínimo de 25 docentes (Ministerio de Educación de Israel, 2019).

Los docentes tienen una capacitación de 24 horas frontales y 6 horas de visitas profesionales. Aquellas instituciones que seguirán en los diferentes niveles de 
profundización del programa recibirán otras 27 a 30 horas de acompañamiento al equipo docente de la institución educativa que lidera este proceso. En el tercer año, este tiempo de acompañamiento se reduce a 15 horas anuales (Ministerio de Educación de Israel, 2012).

De igual manera, se definen siete principios que deben integrarse a un mínimo de cuatro asignaturas en tres niveles diferentes. Esto con el fin de cumplir con el programa y que no sea solamente una capacitación, sino un proceso que la institución continuará por los siguientes años. Además, las instituciones educativas tienen que diseñar un plan para implementar un estilo de vida sostenible, así como un programa para realizar actividades comunitarias sobre los temas que la escuela elija promover (Ministerio de Educación de Israel, 2019).

En Israel existen organizaciones no gubernamentales que participan en el sistema educativo y se encuentran bajo supervisión ministerial (educación inclusiva, educación vial, educación para jóvenes en riesgo, etc.). Dichas organizaciones cumplen una función fundamental, ya que cuentan con la pericia en las áreas que el Ministerio les autoriza a tratar. Los procesos de capacitación y acompańamiento en este programa son liderados por las dos organizaciones nombradas (Red Verde y la Sociedad para la Protección de la Naturaleza). La organización no gubernamental Red Verde es la más importante en relación con la capacitación docente en el área de educación para el desarrollo sostenible. A continuación, se describe sus fortalezas y áreas de trabajo.

\section{Conociendo a Red Verde}

Haciendo referencia a su historia y a su lugar en el sistema educativo israelí, esta organización fue creada en 1998 por el Centro Heschel para la Sostenibilidad. Desde de sus comienzos tuvieron como objetivo incluir su visión de sustentabilidad en la sociedad israelí. Es importante remarcar que Red Verde fue fundada antes de que el programa de Educación para la Sustentabilidad fuese creado y antes de que el concepto de sustentabilidad o educación para la sustentabilidad fuera parte del sistema educativo de Israel. En esa época se trataba exclusivamente de educación para la conservación de la naturaleza. Con los años, este concepto cambió y se transformó 
en lo que conocemos como educación para la sustentabilidad. Según los datos presentados, en la actualidad trabajan con unas 25 autoridades locales en el país, desde el sur del Negev hasta Merom Galilee, y en más de 700 escuelas y jardines de infancia (Red Verde, 2020).

Como se evidencia en su nombre, esta organización tiene principalmente una orientación ambiental, pero cuenta con fuertes bases en temáticas de justicia social. Esto queda ejemplificado cuando Ben Mordecahi y Finger, capacitadoras de la Red Verde, explican acerca de las bases de sus capacitaciones y utilizan los términos justicia social y justicia ambiental como parte significativa de su discurso (A. Mordecahi y E. Finger, comunicación personal, 20 de enero 2020). El concepto de desarrollo sostenible tuvo un proceso dentro de la organización; los temas ambientales se observan y tratan desde una perspectiva integral de desarrollo sostenible. Los ejemplos de las prácticas educativas presentados en los cuestionarios y en gran parte de las entrevistas hacen referencia a temas ambientales.

Como parte de su visión tienen los siguientes principios:

Operar en todos los círculos en Israel: judíos, árabes, beduinos, religiosos y seculares en el centro y la periferia. Consideramos promover el multiculturalismo, fomentar el pluralismo, el respeto y el cuidado del "otro" como parte del concepto de sostenibilidad y propósito de la Red Verde. (Red Verde, 2020)

\section{Metodología}

Uno de los documentos que mejor plantea y presenta el concepto de educación para la paz en Israel es el que presenta los resultados del Comité Público para la Formación de la Política Estatal sobre Educación para una vida conjunta entre judios y árabes en Israel (Salomon e Issawi, 2009). Este contextualiza los conceptos y variables más esenciales y será utilizado como referencia para la investigación. Aquí se presentan una serie de capacidades, habilidades y contenidos que debería ser las bases de la 
práctica educativa, según el Comité para Programas de Educación para la Paz en Israel. Estas capacidades, habilidades y contenidos fueron utilizadas como referencia en la investigación para analizar esta relación. En el anexo 1 se encuentran las listas de capacidades, habilidades y contenidos obtenidos de este documento.

\section{Método de investigación y metodología de análisis}

La primera parte de esta investigación se basó en un análisis de los documentos oficiales del programa de Educación para la Sustentabilidad del Ministerio de Educación en Israel. Esto con el fin de identificar sus principales líneas de trabajo. También se examinó el esquema de la educación para la sostenibilidad bajo el nombre Educación para la sostenibilidad - Tejiendo la vida juntos (Ministerio de Educación de Israel, 2012) y el esquema para el desarrollo profesional, El Programa Integrado de Educación para la Sostenibilidad (Ministerio de Educación de Israel, 2014). En esta primera etapa se entrevistó a la directora del programa en el Ministerio de Educación, con el objetivo de conocer su visión como educadora y la manera como entiende la relación entre los conceptos de desarrollo sostenible y paz.

En la segunda parte de la investigación se verificó el concepto de paz en la práctica del programa de educación para el desarrollo sostenible. Se condujeron encuestas en línea a 19 formadores de docentes y entrevistas semiestructuradas a 4 educadoras, todos de la organización Red Verde. Los 19 formadores pertenecen a los sectores más representativos de la sociedad (judíos religiosos, judíos laicos, árabes musulmanes, árabes cristianos) y en su mayoría son mujeres. Por su parte, las cuatro educadoras entrevistadas tienen diferentes áreas de especialización: historia, educación ambiental, trabajo social, etc., y ocupan cargos de coordinación dentro de la organización.

\section{Acerca del cuestionario sobre capacidades, habilidades y contenidos para educadores}

Para la recolección de la información se condujeron cuestionarios en línea, los cuales fueron diseñados con el objetivo de saber en qué medida los capacitadores 
tenían conocimiento sobre temáticas relacionadas con la educación para la paz. Los cuestionarios fueron resueltos de manera anónima y se dividieron en cuatro partes. Las primeras tres abordaron las capacidades, habilidades y contenidos (anexo 1) que fueron tomados del informe del Comité Público para la Formación de la Politica Estatal sobre Educación para una vida conjunta entre judios y árabes en Israel (Salomon e Issawi, 2009). El Profesor Gavriel Salomon, quien lideró el Comité, fue uno de los referentes más importantes en Israel sobre la educación para la paz. Por esta razón, se seleccionó como precedente para la creación de las categorías —y no otra fuente internacional—, lo que les da coherencia con el contexto.

En estas tres primeras secciones los formadores debían responder en qué medida estas capacidades, habilidades y contenidos eran trabajados en sus capacitaciones con los docentes. Teniendo en cuenta que estos aspectos no se trataban de manera directa en el contexto de la educación para la sostenibilidad — ya que no aparecen en los documentos oficiales del programa-, se agregó un ítem aclaratorio que pedía a los capacitadores dar un ejemplo de la manera que cierta capacidad, habilidad o contenido se trabajaba en las capacitaciones. En la última parte de cuestionario en línea se preguntó, de manera directa, la opinión del capacitador acerca de la relación entre la educación para la paz y la educación para el desarrollo sostenible. Por su parte, en las entrevistas semiestructuradas se indagó con base en las preguntas de los cuestionarios en línea, pero se profundizó en algunos temas que incluían ambas prácticas educativas. Se buscó que los formadores entrevistados representaran a la población de capacitadores, por lo que se incluyeron árabes y judíos.

\section{RESULTADOS Y DISCUSIÓN}

Luego de realizar la lectura y el análisis de los dos documentos oficiales escogidos, que describen y explican el programa de educación para el desarrollo sostenible del Ministerio de Educación de Israel, se evidenció que el concepto de paz no aparece de manera destacada, ni claramente visible. Esto no debe sorprender, ya que las temáticas que hacen referencia a los conceptos paz o conflicto árabe-israeli son delicadas y sensibles para gran parte de la población en el país. Como consecuencia, 
en los documentos sobre los programas nacionales de educación esto se atiende con cuidado. Estos conceptos tienen connotaciones y significados diferentes para los individuos en lugares donde el conflicto está latente (Biton y Salomon, 2006; Bensen Mor, 2018).

$\mathrm{Al}$ realizar un análisis más profundo de los textos del Ministerio de Educación de Israel en el marco de este programa, se distinguió una serie de referencias que no se puede dejar de considerar. En relación con la esencia de la educación para la sostenibilidad, se pueden remarcar los siguientes conceptos básicos: "la sostenibilidad requiere el desarrollo de un conjunto de valores que incluyan valores fundamentales como respeto al medio ambiente, democracia (responsabilidad, participación pública), igualdad (equidad, justicia social y ambiental), paz y aprendizaje permanente" (2012, p. 9). "La educación para la sostenibilidad también es una educación político-cívica para el cambio cultural social, para el desarrollo de la capacidad de participación democrática activa, para el pensamiento crítico sobre la situación existente” (2012, p. 10). Y “[...] una cosmovisión democrática optimista que coloca la dignidad humana y la competencia en el centro de nuestro profundo conocimiento de que todos compartimos el tejido de la vida que permite la existencia de todos los seres en la tierra" (2014, p. 3). De estos fragmentos es posible inferir una serie de conceptos sociales que hace parte de la educación para la paz.

Asimismo, en ambos textos (Ministerio de Educación 2012, 2014) hay una clara alusión a la dimensión social del desarrollo sostenible y una continua referencia de dicha dimensión como pilar del mismo. Esto tiene que ver con los orígenes del programa y sus ancestros en el sistema educativo israelí, como ya fue explicado.

Para analizar en profundidad estos textos, se exploraron los conceptos (anexo 1) presentados por Salomon e Issawi (2009) para poder ver su relevancia en los documentos escogidos.

En cuanto a las capacidades, queda claro que el pensamiento critico-reflexivo es una de las más habituales en las prácticas educativas de este programa. Esto se observa en la frecuencia que aparece este concepto en los documentos oficiales del Ministerio. 
En la práctica, según lo explicado en las entrevistas, esta capacidad se desarrolla a través del análisis de casos lejanos o propios, pero del pasado. Ben Mordecahi, capacitadora de Red Verde, cuenta que utiliza ejemplos de problemas medioambientales de otras partes del mundo y la manera como se resuelven o en qué situación se encuentran. Es importante destacar, que no utiliza la palabra conflicto, sino problema (A. Mordecahi, comunicación personal, 20 de enero 2020). Por su parte, Finger, también capacitadora de Red Verde, acude a ejemplos de la comunidad, pero de otras épocas, para así hacer la comparación con el comportamiento actual (E. Finger, comunicación personal, 20 de enero 2020). Kupermintz y Salomon (2005) resaltan la importancia de esta práctica en lugares donde los conflictos están presentes y son continuos. Esta es llamada high road transfer y hace referencia a una abstracción consciente, con el objetivo de transferir las conclusiones del caso ajeno a su realidad cercana.

Otras dos capacidades que se resaltan en estos documentos son la cooperación y la resolución de problemas. Estas tienen relación con la capacidad de resolución de conflictos, la cual está ligada a la pregunta que aparece en los cuestionarios en línea: ¿qué capacidades se desarrollan en las capacitaciones? Es importante aclarar que en los cuestionarios en línea esta recibió un valor bajo. Esto está relacionado con el hecho de que la pregunta incluía la palabra conflicto, lo cual hace referencia directa al conflicto político existente en el país y genera antagonismo, como se pudo ilustrar mediante las respuestas de los capacitadores.

A pesar de que los conceptos empatía y tolerancia no aparecen de manera directa y repetida en gran parte de los textos, sí se encuentran los otros y consideración por las necesidades. Además, debido a que el programa funciona en instituciones educativas árabes e israelíes, en las entrevistas con las capacitadoras sí se mencionaron estos conceptos. Para aquellas capacitadoras que trabajan con diferentes poblaciones o solamente con poblaciones árabes estos temas están presentes.

En relación con las habilidades que aparecen en el cuestionario, en los documentos del Ministerio no se presentan de manera similar, ya que de las siete habilidades, cuatro tienen vínculo con la palabra conflicto. Esto genera un antagonismo significativo, como se explicó, y por ello no se utiliza. 
Las habilidades que se encuentran en los documentos del ministerio y que tienen relación con las del cuestionario son:

- Pensamiento sistémico

- Pensamiento crítico

- Comunicación y colaboración

- Resolución de problemas y toma de decisiones

- Toma pequeña posición

- Comunicación interpersonal y social como parte de la cultura del discurso y la discusión

- Conciencia para la colaboración

- Trabajo en equipo

- Integración en una comunidad en línea (Ministerio de Educación de Israel, 2012, pp. 12, 23 y 70).

En lo que se refiere a los contenidos, hay espacio para la interpretación. De todas formas, contenidos como democracia, igualdad o derechos prácticamente no tienen mucho espacio para ser interpretados de maneras diferentes y es necesario entender si aparecen o no en estos documentos. En el caso de democracia e igualdad, ambos conceptos aparecen repetidamente, mientras que derechos en cualquiera de sus versiones (del niño, del estudiante, de los animales, etc.) no se evidencia con la misma frecuencia. Por otra parte, las palabras poblaciones y cultura, aparecen repetidamente, lo que apunta a que sí se hace referencia a la diversidad de la población. 
El concepto ciudadanía puede ser interpretado de diferentes formas e incluso conectarse al campo de los derechos civiles. En estos documentos queda claro que la referencia está hecha mayoritariamente al activismo y acción del individuo frente a los problemas en la realidad social y ambiental.

Después de realizar la lectura de los documentos oficiales, el siguiente paso fue comparar los resultados de los cuestionarios en línea y las entrevistas. En la figura 1 se pueden ver las respuestas de los 19 capacitadores de Red Verde a la primera pregunta.

Figura 1. Respuestas de los capacitadores de Red Verde a la primera pregunta

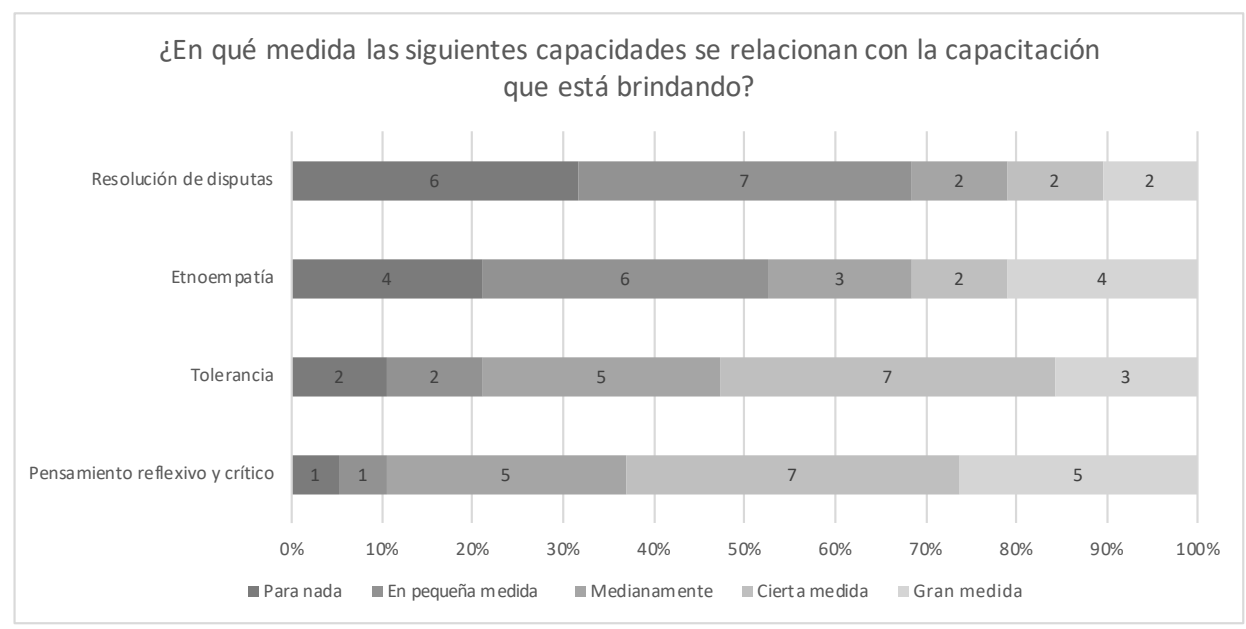

Fuente: elaboración propia.

Las respuestas de los educadores siguen la misma línea que presentan los documentos oficiales en relación con la relevancia de cada una de las capacidades. Se evidencia claramente que las dos primeras, pensamiento reflexivo y crítico y tolerancia, están presentes con mayor frecuencia en sus capacitaciones. Por otro lado, la etnoempatía y resolución de disputas reciben valores menores en cuanto a su presencia en las sesiones de formación. Sobre esta última capacidad, nuevamente se observa que la palabra disputas, que en hebreo se traduce también como conflicto, recibe notoriamente valores menores. 
La mayoría de los ejemplos propuestos hacen referencia al medio ambiente y a la capacidad de pensamiento crítico y reflexivo, y etnoempatía. Estos son las más resaltadas al momento de ejemplificar. En relación con la capacidad de tolerancia, esta se manifestó a través del trabajo que realizan los docentes durante la capacitación y no mediante los contenidos o las temáticas del programa.

En la figura 2 se aprecian las respuestas a la segunda pregunta. Como ya se mencionó, esta fue la que evidenció el antagonismo frente a la palabra conflicto.

Figura 2. Respuestas de los capacitadores a la segunda pregunta

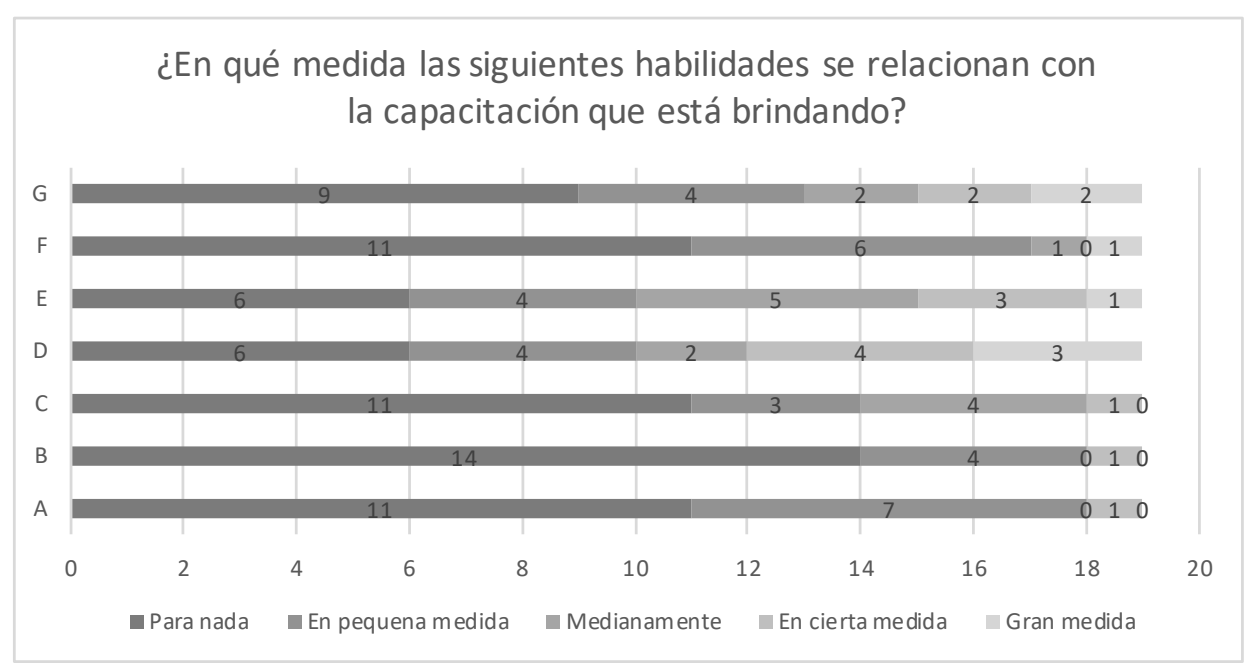

Fuente: elaboración propia.

A continuación, se presentan las siete habilidades del cuestionario en línea:

1. Comprender que el conflicto es una parte necesaria y natural de la vida.

2. Desarrollar habilidades de gestión de conflictos: saber qué enfoque para la resolución pacífica de conflictos es mejor para un problema de conflicto específico. 
3. Desarrollar una conciencia vital para comprender la perspectiva de la otra parte y liderar el proceso de resolución del conflicto de manera constructiva.

4. Desarrollar la capacidad de distinguir actitudes de necesidades o intereses.

5. Fomentar la capacidad de expresar emociones de manera no agresiva.

6. Desarrollar la capacidad para definir el conflicto como un problema mutuo que debe resolverse de manera colaborativa mientras se llega a un compromiso a través de la negociación y/o con la ayuda de un tercero.

7. Realizar una lluvia de ideas que creará, refinará y mejorará la variedad de soluciones pacíficas.

Como se explicó, la palabra conflicto despierta un antagonismo que queda claro cuando observamos las respuestas proporcionadas. Es importante remarcar que el enunciado en hebreo hace referencia al conflicto de manera genérica y no a uno específico, como se podría interpretar del enunciado en español.

Dos respuestas sobresalen por motivos diferentes. La primera: "no tratamos directamente el tema de la paz y el conflicto". El problema de este comentario radica en el hecho de que en ninguna instancia se hace referencia al conflicto árabe-israelí, ni se especifica conflicto alguno. Esto nuevamente refuerza la referencia que paz y conflicto tienen entre los capacitadores. Aquellos que lograron desconectar el conflicto político existente en el país de los conceptos conflictos y paz, como miembros de una sociedad, mencionaron ejemplos ambientales con roces económicos y de "paz con uno mismo y el entorno". Estos comentarios también se conectan con el fuerte componente ambiental del programa.

La figura 3 corresponde a una serie de contenidos (anexo 1) que, en las conclusiones del Comité, aparecen como centrales a la hora de diseñar un programa de educación para la paz. Los contenidos presentados se podrían clasificar como: propios, compartidos e interpretables. Los propios corresponden a aquellos que difícilmente se 
podrían encontrar en otros programas educativos; es decir, que son propios de esta práctica. Los compartidos serían aquellos que se pueden encontrar en otros programas educativos. Finalmente, los interpretables son aquellos que el contenido en sí mismo puede ser embarcado de diferentes formas.

De esta manera, vemos que los contenidos compartidos - como la democracia, los derechos humanos, la igualdad y la diversidad— son los que tienen una calificación alta. Los contenidos propios, que al mismo tiempo son menos interpretables, califican bajos. Algunos ejemplos de estos contenidos son: estereotipos, derechos de minorias y disputas (o conflictos). Finalmente, contenidos como relaciones entre mayoria y minoría e identidad son interpretables de diferentes formas, por eso no son excluidos, pero no califican alto.

Figura 3. Contenidos centrales para diseñar un programa de educación para la paz

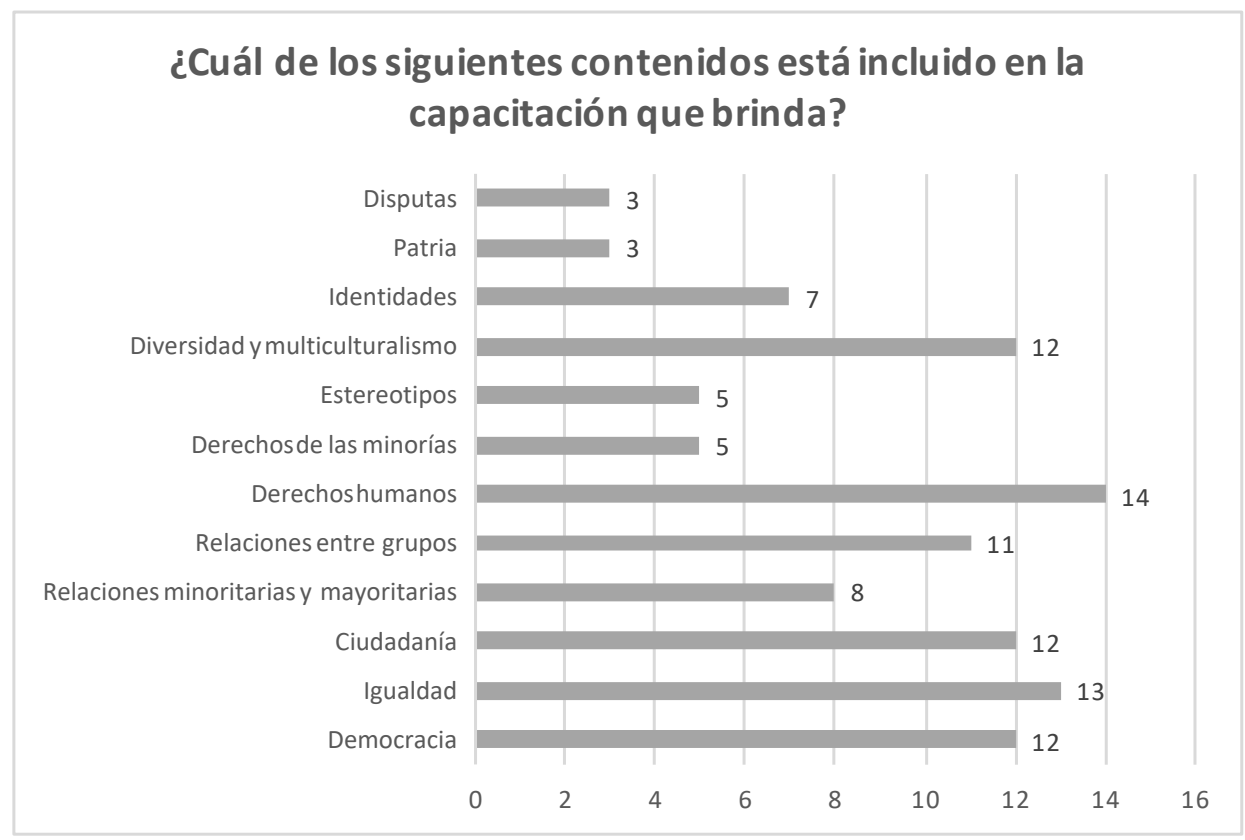

Fuente: elaboración propia con base en el Comité Público para la Formación de la Política Estatal sobre Educación para una vida conjunta entre judíos y árabes en Israel (Salomon e Issawi, 2009). 
En la última sección de los cuestionarios se presenta una serie de puntos directamente relacionados con la pregunta de investigación, y sus respuestas muestran la opinión de los capacitadores de manera directa y descriptiva. A la pregunta: ¡existe alguna relación entre estos dos practicas educativas?, la respuesta fue afirmativa y prácticamente unánime, con una única respuesta negativa. Los capacitadores lograron describir de manera teórica esta relación, haciendo énfasis en los valores e ideas que complementan estas prácticas educativas. A continuación, se presentan loscomentarios de los capacitadores 1, 6, 12 y 14, obtenidos de los cuestionarios anónimos en línea realizados a la Red Verde.

- “Ambas son prácticas educativas para ver cómo podemos usar lo que existe y dejar un buen mundo a quienes nos siguen" (comunicación personal, 26 de diciembre de 2019).

- "La justicia ambiental está asociada con la justicia social, y es el motivo de la educación para la paz” (comunicación personal, 26 de diciembre de 2019).

- "En mi opinión, un concepto básico de sostenibilidad es la percepción de las relaciones y los derechos entre las personas. Personalmente, no puedo ver que la sostenibilidad vaya con el racismo. Sus valores sociales y justicia social están en la raíz de las percepciones de sostenibilidad" (comunicación personal, 29 de diciembre de 2019).

- "Existe un enfoque para comprender la forma en que existe reciprocidad y dignidad entre el hombre y la tierra, y el hombre y el hombre" (comunicación personal, 29 de diciembre de 2019).

Desde una perspectiva más crítica sobre la situación de la educación para la paz en el sistema educativo israelí y específicamente sobre el programa de educación para la sustentabilidad, aparecen algunas incógnitas.

En el Ministerio de Educación de Israel no existe un programa que esté centrado en la educación para la paz. El autor Meruyak Klerman (2011) cuestiona el lugar que se le da a esta práctica en el Ministerio de Educación y aborda la importancia de trabajar estos contenidos de manera individual e integrada en los programas de 
estudio. En la Secretaría Pedagógica se encuentra el programa de Educación cívica y para la convivencia, el cual hace referencia directa a conceptos y temáticas que tiene que ver con la educación para la paz. De todas maneras, solo parte de las capacidades, habilidades y contenidos incluidos en la investigación aparece en este programa, lo que no da respuesta a la crítica de Meruyak Klerman (2011).

Algunas de las respuestas anónimas de los capacitadores de Red Verde en los cuestionarios en línea llaman a la reflexión. A continuación, los comentarios de los capacitadores 4 y 14:

- "No tratamos directamente el tema de paz y el conflicto" (comunicación personal, 26 de diciembre de 2019).

- “[...] no es mi mandato como facilitador para la sostenibilidad introducir esto en mi trabajo" (comunicación personal, 29 de diciembre de 2019).

La educación para el desarrollo sostenible apunta a un cambio de conducta y esto puede generar conflictos. Evitar su relevancia al tratar esta temática es no tratar todo el proceso educativo. Sería interesante preguntarles a estos capacitadores cómo entienden los conflictos relacionados con las temáticas de desarrollo sostenible como parte de su mandato.

Si tomamos la dimensión ambiental como ejemplo, comprendemos que esta vive en conflicto con la conducta humana. En estas prácticas educativas se intenta generar una concientización, una recapacitación sobre la conducta humana que en las últimas décadas logró probar su poder destructivo sobre el entorno. El daño ambiental genera conflictos de índole político e incluso guerras. Las disputas generadas entre seres humanos - y no solamente para con la naturaleza - son difícilmente negables y parte integral de la realidad (Harris, 2004; Bajaj y Chiu, 2009).

Llevemos lo antes presentado al aula de clase. En el caso de que un docente quiere conversar en su clase sobre la decisión de un gobierno o ente público frente a un recurso natural local que causa su degradación o destrucción, puede plantear la siguiente pregunta a sus estudiantes: ¿qué sugieren que se debe hacer? Las respuestas 
a este tipo de preguntas lo llevarán a áreas relacionadas con violencia, resolución de conflictos, democracias, justicias, empatía, el otro, etc. Estos son conceptos básicos de la educación para la paz (Harris, 2004; Bensen Mor, 2018).

\section{Diferenciando la conceptualización de paz en el contexto israelí}

La manera en que el concepto de paz es comprendido varía dependiendo del contexto y la sociedad. Esto tiene un efecto directo en cómo se plantea y qué impacto tienen las prácticas de educación para la paz en las diferentes partes del mundo. No todos los contextos son iguales y, por ende, no siempre son comparables (Salomon, 2004; Biron y Salomon, 2006; Bensen Mor, 2018).

De los resultados presentados y las entrevistas realizadas, queda claro que en el contexto israelí la palabra conflicto despierta antagonismo y hace alusión al conflicto político. En los documentos del Ministerio se hace referencia a problemas y no a conflictos. En general, y en hebreo específicamente, el concepto problemas no genera una relación con un conflicto, sino con una situación. En el momento en que una situación o problema se define como conflicto, automáticamente hay lados y como queda claro que esto se quiere evitar.

En el caso del conflicto árabe-israelí existen dos particularidades que vale la pena resaltar. En primer lugar, el conflicto existente no es entre individuos, sino entre colectivos. La relación de un ciudadano judío-israelí y un ciudadano árabe-israelí o palestino en la autoridad palestina constituye, en gran medida, una reflexión del conflicto entre colectivos, entre sus intereses e ideas, y no entre los individuos. En segundo lugar, el caso israelí-palestino es un conflicto continuo, con muchos años de historia y sumamente latente en el día a día del país. Por ello, nuestra situación es comprable —en cierta medida y en periodos específicos - con Kosovo, Chipre o Irlanda del Norte. Por el contrario, en Estados Unidos de América, Japón u otros países, la educación para la paz puede estar relacionada con tensiones con minorías, como los inmigrantes, o hechos del pasado. En ambos contextos se puede justificar esta práctica, pero los objetivos y acciones serán diferentes (Salomon, 2004; Kupermintz y Salomon, 2005; Bensen Mor, 2018). 
Es en este contexto, las prácticas clásicas que se dan en estos programas, como proyectos o encuentros entre individuos, son menos efectivas para cambiar la perspectiva de los colectivos. Esto se destaca en las investigaciones realizadas por Salomon (2004) y Biton y Salomon (2006), en las que se analizan este tipo de actividades y su impacto. Asimismo, en una de las entrevistas realizadas en la investigación, la representante de Red Verde menciona que ella tampoco cree en este tipo de estrategias, sino en procesos del diario vivir y a largo plazo que no se focalizan en llegar a la paz como concepto, sino en comprender las necesidades y rutinas de las comunidades (R. Akel, comunicación personal, 19 enero 2020).

De lo anterior surge la pregunta: ¿con qué objetivo se realizan estas prácticas? El doctor Haggai Kupermintz, de la Universidad de Haifa, explica, en la entrevista realizada en el 2007, que la importancia de estas actividades en situaciones en las que los conflictos son continuos y hacen parte integral de la rutina, logran prevenir el deterioro constante de las percepciones y sentimientos entre los grupos (Universidad de Haifa, 2007). Además, en las entrevistas realizadas a tres de las capacitadoras de Red Verde, se resalta que, en sus prácticas, ellas sí abordan la temática del otro y sí trabajan con ejemplos de situaciones en otros contextos con el fin de sensibilizar a los participantes (Yael Barki, Ayelet Ben Mordecahi y Efrat Yael Finger, comunicación personal, 20 de enero 2020). Como se explicó, esto busca una sensibilización a través del análisis crítico-reflexivo que genere una abstracción consciente que sea transferible (Kupermintz y Salomon, 2005).

La validez interna de esta investigación es sobresaliente, considerando que Red Verde es uno de los dos organismos encargados de la capacitación de los educadores israelíes en servicio, en esta materia. Dentro de Red Verde participaron $75 \%$ de los capacitadores y el $100 \%$ de estos trabajan con educación primaria y secundaria.

En cuanto a su validez externa, los datos obtenidos son más relativos. Por un lado, las referencias internacionales son las mismas; por ello, el punto de partida de otros ministerios de educación en otros países que utilizan este referente puede ser similar. Por otro lado, las similitudes en los programas de educación para la sustentabilidad en otros países deben ser investigadas. Además, se debe analizar a profundidad el contexto nacional para poder conocer las connotaciones que tienen los conceptos de paz y conflicto. 


\section{Conclusiones}

Las preguntas que se deben responder son: ¿qué tipo de relación comparten estas prácticas en el programa de educación para la sustentabilidad en el contexto israelí? ¿Se trata de una relación visible o invisible? Cuando se hizo alusión a los programas educativos específicos dentro del sistema de educación israelí, se encontraron relaciones directas e indirectas haciendo uso de palabras clave.

En relación con la práctica educativa, se quiso entender de facto qué actividades se realizan con los estudiantes. Durante las entrevistas se encontraron ejemplos de prácticas que son similares en la educación para la paz y la educación para la sustentabilidad. Lo interesante aquí fue que, al aplicar los cuestionarios e indagar de manera genérica — tomando como referencia la educación para la paz- las respuestas fueron diferentes. Esta cuasicontradicción fortalece la relación con lo explicado anteriormente en relación con la palabra conflicto. Los conceptos paz y conflicto tienen una connotación delicada, por no describirla como negativa. Este antagonismo se percibió en los cuestionarios en línea y se acentuó en las entrevistas. Por otro lado, la falta de conocimiento por parte de los capacitadores sobre el tema también genera dificultades a la hora de conectar ambas prácticas bajo títulos y descripciones genéricas de habilidades o capacidades.

De las entrevistas y los cuestionarios queda claro que, aunque los contenidos clásicos de la educación para la paz — como los derechos del individuo, los conceptos de patria, estereotipos o el significado y tipo de conflictos-, tienen un menor espacio en las capacitaciones, sí se busca desarrollar habilidades y capacidades relacionadas con la educación para la paz. Asimismo, los capacitadores no reconocieron que las capacidades, habilidades o contenidos sí pueden ser compartidas o relacionadas como parte de la educación para la paz. En la actualidad, se le otorga un lugar más sobresaliente a las habilidades y las capacidades, ya que estas son fundamentales para la formación de los estudiantes en contextos tan versátiles y cambiantes. Esto quiere decir que, aunque los capacitadores no las reconozcan en sus prácticas, existen una serie de capacidades, habilidades y contenidos que sí se están impartiendo, lo cual resulta importante. Esto es lógico considerando que, en más de una oportunidad, los capacitadores expresaron su falta de conocimiento sobre el tema. 
En la entrevista con Hani Peleg, directora del Programa de Educación para la Sustentabilidad del Ministerio de Educación, uno de los puntos que destacó fue que el programa no está politizado. Esto quiere decir que no está conectado ni con políticas específicas, ni con el conflicto político existente o algún grupo específico de la sociedad. Esto lo hace atractivo y accesible para todas las escuelas, tanto judías, musulmanes, cristianas, laicas y religiosas (Hani Peleg comunicación personal, 28 de diciembre de 2019). Además, este es un programa que se integra en toda la institución y en su rutina, lo que debería generar cambios en los comportamientos de los docentes y estudiantes.

Este programa tiene condiciones para generar logros muy significativos, ya que cuenta con una serie de mensajes innatos. Sería correcto que la educación para la paz sí hiciera parte de la planificación estratégica del programa —integrando de manera más significativa las habilidades y capacidades-, pero no como una característica visible de este. En un país donde los conceptos de paz y conflicto generan tanto antagonismo, rechazo y discusión, quizá lo correcto es de manera no visible nutrir a las nuevas generaciones con estas habilidades y capacidades para tener un mejor futuro. Es posible que este programa sea una herramienta invisible de la educación para la paz, pero esto se conocerá en ańos venideros.

\section{REFERENCIAS}

Agudelo, M. G. (2018). Educación para el desarrollo sostenible. Una mirada a los proyectos ambientales escolares Prae. Libre Empresa, 15(2), 179-194. Dor: https://doi. org/10.18041/1657-2815/libreempresa.2018v15n2.5360

Bajaj, M. y Chiu, B. (2009). Education for sustainable development as peace education. Peace \& Change, 34(4), 441-455. Dor: https://doi.org/10.1111/j.1468-0130.2009.00593.x

Bensen Mor, N. (2018). Educación para la paz. Lexi-Kaye, (9), 10-12. Recuperado de https://

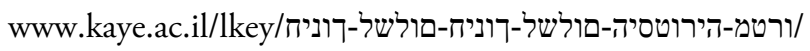


Biton, Y. y Salomon, G. (2006). Peace in the eyes of Israeli and Palestinian youths: Effects of collective narratives and peace education program. Journal of Peace Research, 43(2), 167-180. DoI: https://doi.org/10.1177/0022343306061888

Goldman, D., Yavetz, B. y Pe'er, S. (2006). Environmental literacy in teacher training in Israel: Environmental behavior of new students. The Journal of Environmental Education, 38(1), 3-22. DoI: https://doi.org/10.3200/JOEE.38.1.3-22

Filut, D. (2018). Conceptualización y caracterización de la educación hacia el desarrollo sostenible. En L. Albor-Chadid (ed.), Educación socio-ambiental. Acción-Presente (pp. 13-44). Barranquilla: Universidad Simón Bolívar.

Harris, I. M. (2004). Peace education theory. Journal of Peace Education, 1(1), 5-20. DoI: https://doi.org/10.1080/1740020032000178276

Kupermintz, H. y Salomon, G. (2005). Lessons to be learned from research on peace education in the context of intractable conflict. Theory into practice, 44(4), 293-302. DOI: https://doi.org/10.1207/s15430421 tip4404_3

Meruyak Klerman, O. (2011). ¿Qué es la educación para la paz y cómo se puede promover? (en hebreo). The Adam institute for Democracy and Peace. Recuperado de http://www. adaminstitute.org.il/wp-content/uploads/2015/06/מדקל-רשפא-דציכו-םולשל-דוניח-והמ.pdf

Ministerio de Educación de Israel. (2012). Educación para la sostenibilidad-Tejiendo la vida juntos (en hebreo). Recuperado de http://meyda.education.gov.il/files/Mazkirut_Pedagogit/MadaTechnologya/sustainableeduyesodi[1].pdf

Ministerio de Educación de Israel. (2014). El Programa Integrado de Educación para la Sostenibilidad (en hebreo). Recuperado de http://meyda.education.gov.il/files/Mazkirut_ Pedagogit/MadaTechnologya/tohnit_meshulevet_hinuh_kayamut_11_03_2015.pdf

Ministerio de Educación de Israel (2019). Programas de educación para la sostenibilidad: programa integrado para implementar la educación para la sostenibilidad en el sistema educativo (en hebreo). Recuperado de http://cms.education.gov.il/EducationCMS/Units/ Mazkirut_Pedagogit/MadaTechnologya/kayamut/tochniyot/tochniut.htm 
Naciones Unidas. (1945). Carta de las Naciones Unidas. Recuperado de https://www.un.org/ es/charter-united-nations/index.html.

Naciones Unidas. (2015). Transformar nuestro mundo: la Agenda 2030 para el Desarrollo Sostenible. Recuperado de https://unctad.org/meetings/es/SessionalDocuments/ ares70d1_es.pdf.

Salomon, G. (2004). Does peace education make a difference in the context of an intractable conflict? Peace and Conflict, 10(3), 257-274. Dor: https://doi.org/10.1207/ s15327949pac1003_3

Salomon, G. (2006). Does peace education really make a difference? Peace and Conflict, 12(1), 37-48. DoI: https://doi.org/10.1207/s15327949pac1201_3

Salomon, G. e Issawi, M. (2009). Comité Público para la Formación de la Política Estatal sobre Educación para una vida conjunta entre judíos y árabes en Israel (en hebreo). Recuperado de https://www.news1.co.il/uploadFiles/246196925640107.pdf

Surendra, L. (2014). The Role of Education in Promoting Sustainable Development and Peace. Education for Peace and Sustainable Development, 25. Recuperado el 26 de marzo de 2020. Disponible en http://in.one.un.org/wp-content/uploads/2016/09/227521e. pdf\#page $=25$

Red Verde (s. f.). Historia. Recuperado de http://www.reshet-yeruka.net/הירוטסיה/ונחנא-ימ/

Universidad de Haifa. (31 de enero de 2007). ¿Para quése necesita la educación para la paz?

[Video] Recuperado de https://www.youtube.com/watch?v=RWuxTvw-6bk\&t=342s

World Commission on Environment and Development (WCED) (1987). Our common future. The Brundtland Report. Oxford: Oxford University Press. 
Anexo 1. Capacidades, habilidades y contenidos obtenidos del documento Comité Público para la Formación de la Politica Estatal sobre Educación para una vida conjunta entre judios y árabes en Israel (Salomon e Issawi, 2009)

\section{Capacidades}

- Pensamiento reflexivo y crítico

- Tolerancia

- Etnoempatía

- Resolución de disputas

\section{Habilidades}

- Comprender que el conflicto es una parte necesaria y natural de la vida.

- Desarrollar habilidades de gestión de conflictos: saber qué enfoque para la resolución pacífica de conflictos es mejor para un problema de conflicto específico.

- Desarrollar una conciencia vital para comprender la perspectiva de la otra parte y liderar el proceso de resolución del conflicto de manera constructiva.

- Desarrollar la capacidad de distinguir actitudes de necesidades o intereses.

- Fomentar la capacidad de expresar emociones de manera no agresiva.

- Desarrollar la capacidad para definir el conflicto como un problema mutuo que debe resolverse de manera colaborativa mientras se llega a un compromiso a través de la negociación y/o con la ayuda de un tercero.

- Realizar una lluvia de ideas que creará, refinará y mejorará la variedad de soluciones pacíficas.

\section{Contenidos}

- Democracia

- Igualdad

- Ciudadanía

- Relaciones minoritarias y mayoritarias

- Relaciones entre grupos

- Derechos humanos

- Derechos de las minorías

- Estereotipos

- Diversidad y multiculturalismo

- Identidades

- Patria

- Disputas 